\section{Tromboprofilaxia primária}

A profilaxia primária de TEV tem sido recomendada fartamente na literatura médica e baseia-se na alta incidência de TVP e/ou TEP em pacientes hospitalizados. ${ }^{(1,2)}$ Apesar de um grande número de estudos comprovarem a eficácia da tromboprofilaxia, essa ainda é muito subutilizada, tanto em contextos cirúrgicos, quanto clínicos. ${ }^{(3)}$

Uma alternativa à profilaxia seria a realização de exames de membros inferiores em pacientes de alto risco de TEV; entretanto, a ultrassonografia Doppler venosa tem sensibilidade apenas moderada para diagnosticar TVP em pacientes assintomáticos. ${ }^{(4)}$ Essa estratégia não demonstrou ser custo-efetiva e, portanto, não é recomendada.(B) ${ }^{(5)}$

0 risco de TEV varia conforme o cenário do paciente, isto é, conforme a presença de fatores de risco inerentes ao paciente e ao procedimento, no caso dos pacientes cirúrgicos. É fundamental que todo paciente hospitalizado tenha seu risco estimado e receba a profilaxia para o nível correspondente. $(A)^{(5)}$ Pacientes do grupo de maior risco apresentam uma incidência de TVP distal de 40-80\%; de TVP proximal, de 10-20\%; de TEP, de 4-10\%; e de TEP fatal, de $0,2-5,0 \%$. Dessa forma, necessitam receber medidas preventivas mais intensas que aqueles com risco moderado. ${ }^{(6)}$ Nos Quadros 15 e 16, estão apresentadas as incidências estimadas de TEV nos vários contextos clínicos e, no Quadro 17, a estratificação de risco de TEV com a tromboprofilaxia sugerida.

As medidas disponíveis para a profilaxia da TEV são divididas em métodos mecânicos e farmacológicos. ${ }^{(5)}$ Entretanto, a deambulação precoce e frequente, quando possivel, deve ser sempre estimulada. Um dos métodos mecânicos são as MECG, que são recomendadas para pacientes com risco moderado de TEV,e com tendência elevada de sangramento.(A) ${ }^{(7)} \mathrm{A} C P \mathrm{Cl}$ é o método mecânico sugerido para pacientes com alto risco de TEV, mas também de sangramento.(A) ${ }^{(8)}$ Em cirurgias ortopédicas, também há alguns estudos com as bombas pneumáticas para pés. ${ }^{(9)}$ Os métodos mecânicos também podem ser usados em associação a medidas farmacológicas em pacientes com alto risco de TEV.(A) ${ }^{(10)}$ Os $\mathrm{FVCl}$, sobretudo os temporários, podem excepcionalmente ser empregados na prevenção de TEV, em situações de extremo risco de TEV e sangramento, mas são pouco disponíveis e de alto custo.(C) $)^{(11)}$

A tromboprofilaxia farmacológica incorpora as heparinas (HNF e HBPM), o fondaparinux (pentassacarídeo ainda não comercializado no Brasil), os AVK e, mais recentemente, a dabigatrana (inibidor da trombina). A aspirina não deve ser utilizada como medida isolada para a prevenção de TEV.(A) $)^{(12)}$

0 uso de HNF como profilaxia de TEV é recomendado nos EUA desde o Consenso do National Institute of Health (1986). ${ }^{(13)}$ A HNF reduz em cerca de 70\% a incidência de TEV, em comparação aos pacientes que não usam nenhum tipo de profilaxia. ${ }^{(14)}$ É utilizada nas doses de $5.000 \mathrm{Ul}$, duas ou três vezes ao dia, sendo essa última mais eficaz. ${ }^{(15)}$

As HBPM são tão eficazes quanto à HNF. Meta-análises mostraram que as heparinas reduzem significativamente a incidência de TEV, mas não alteram a mortalidade, sendo que uma dessas meta-análises mostrou uma tendência de maior eficácia para as HBPM. ${ }^{(16,17)} \mathrm{Na}$ escolha da tromboprofilaxia farmacológica (principalmente das HBPM e do fondaparinux), deve ser levada em conta a presença de insuficiência renal, sobretudo em idosos com diabete melito e risco de sangramento. ${ }^{(5)}$

Os AVK, com dose ajustada para manter o $\mathrm{RNI}$, são altamente eficazes na prevenção de TEV, inclusive em situações de alto risco, como cirurgias ortopédicas de quadril ou joelho, apesar de aumentarem o risco de hematoma na ferida operatória. ${ }^{(18)} \mathrm{O}$ AVK deve ser iniciado na noite anterior à cirurgia, e o nível terapêutico é atingido após o terceiro dia pós-operatório.

Qadro 15 - Incidência estimada de TVP em vários grupos de pacientes que não recebiam tromboprofilaxia.

\begin{tabular}{|lc|}
\hline \multicolumn{1}{|c|}{ Contexto } & $\begin{array}{c}\text { Prevalência de } \\
\text { TVP distal, \% }\end{array}$ \\
\hline Pacientes clínicos & $10-20$ \\
Cirurgia geral & $15-40$ \\
Cirurgia ginecológica & $15-40$ \\
Cirurgia urológica maior & $15-40$ \\
Neurocirurgia & $15-40$ \\
AVC & $20-50$ \\
Artroplastia de joelho ou quadril & $40-60$ \\
Politraumatismo & $40-80$ \\
Pacientes críticos & $10-80$ \\
Lesão da medula espinhal & $60-80$ \\
\hline
\end{tabular}

Adaptado do estudo de Geerts et al. ${ }^{(5)}$ 
Quadro 16 - Níveis de risco de TEV e respectivos regimes profiláticos.

\begin{tabular}{|c|c|c|}
\hline Níveis de risco & $\begin{array}{l}\text { Incidência de TVP } \\
\text { distal sem profilaxia }\end{array}$ & Tromboprofilaxia sugerida \\
\hline Risco baixo & \multirow{3}{*}{$<10 \%$} & Tromboprofilaxia farmacológica não \\
\hline - Cirurgia menor que permite deambulação & & recomendada \\
\hline - Paciente clínico que deambula plenamente & & Deambulação precoce e “agressiva” \\
\hline Risco moderado & \multirow{3}{*}{$10-40 \%$} & HBPM, HNF duas ou três vezes ao \\
\hline $\begin{array}{l}\text { - Maioria das cirurgias, cirurgia ginecológica } \\
\text { aberta, urológica, }\end{array}$ & & $\mathrm{dia}^{\mathrm{a}}$ \\
\hline \multicolumn{2}{|l|}{ • Paciente clínico acamado ou prostrado } & \\
\hline $\begin{array}{l}\text { TEV com risco moderado, mas com alto risco de } \\
\text { sangramento }\end{array}$ & & Tromboprofilaxia mecânica (MECG) \\
\hline Risco elevado & & HBPM, cumarínicos $(\mathrm{RNI}=2-3)^{\mathrm{a}}$ \\
\hline $\begin{array}{l}\text { - Artroplastia do quadril ou de joelho, cirurgia } \\
\text { de fratura de quadril }\end{array}$ & \multirow[t]{3}{*}{$40-80 \%$} & \\
\hline • Politraumatismo, lesão da medula espinhal & & \\
\hline $\begin{array}{l}\text { Risco elevado de trombose e também de } \\
\text { sangramento }\end{array}$ & & Tromboprofilaxia mecânica (CPI) \\
\hline
\end{tabular}

${ }^{a}$ s.c. Adaptado do estudo de Geerts et al. ${ }^{(5)}$

O fondaparinux sódico tem sido amplamente estudado em pacientes cirúrgicos e clínicos, mostrando equivalência ou superioridade em relação às heparinas. Não é ainda comercializado no Brasil (mas possui registro na ANVISA) e é recomendado como uma alternativa na prevenção de TEV na maioria das diretrizes internacionais, tendo forte evidência favorável. ${ }^{(5)}$ A dose profilática recomendada é de $2,5 \mathrm{mg} / \mathrm{dia}$ por administração s.c.

0 uso da profilaxia farmacológica com HNF, HBPM ou AVK deve ser empregado em pacientes com risco moderado ou alto de TEV, (A) sendo o uso do fármaco específico escolhido de acordo com as características do paciente e do cenário nosológico.

\section{Profilaxia de TEV em pacientes cirúrgicos}

0 uso da tromboprofilaxia em pacientes cirúrgicos é determinado por uma combinação de fatores inerentes ao paciente e ao tipo e duração da cirurgia. Sua eficácia é evidenciada por um grande número de estudos clínicos. Grandes séries de estudos e várias meta-análises, comparando HNF e HBPM na tromboprofilaxia de pacientes submetidos à cirurgia geral, demonstram que ambas são igualmente seguras e efetivas. ${ }^{(19)}$ As HBPM, entretanto, podem ser administradas em dose única diária e apresentam menor incidência de $\mathrm{TlH}^{\left({ }^{(20)}\right.}$ No Quadro 17, estão apresentadas as doses recomendadas das hepa- rinas para a prevenção de TEV em pacientes cirúrgicos.

Pacientes cirúrgicos com baixo risco de TEV não necessitam receber medidas profiláticas, exceto a deambulação precoce.(A) Já pacientes com risco moderado ou alto devem receber tromboprofilaxia farmacológica e/ou mecânica conforme o nível de risco.(A)

Cirurgias neurológicas maiores requerem profilaxia para TEV. Os métodos mecânicos CPI, com ou sem MCEG, são os mais recomendados devido ao temor de sangramentos intracranianos ou espinais.(A) Em pacientes com maior risco, recomenda-se a combinação de métodos mecânicos e farmacológicos.(B) ${ }^{(21)}$

Pacientes submetidos à cirurgia por neoplasia constituem uma população de alto risco de desenvolver TEV. ${ }^{(22)}$ Um estudo comparou o uso de enoxaparina (40mg, uma vez ao dia) com HNF (5.000 Ul a cada $8 \mathrm{~h}$ ), usadas na profi-

Quadro 17 - Posologia das heparinas para o regime profilático em pacientes cirúrgicos. ${ }^{\text {a }}$

\begin{tabular}{|lcc|}
\hline Fármacos & Risco moderado & Risco alto \\
\hline Dalteparina & $2.500 \mathrm{Ul} /$ dia & $5.000 \mathrm{UI} /$ dia \\
Enoxaparina & $20 \mathrm{mg} /$ dia & $\begin{array}{c}40 \mathrm{mg} / \text { dia ou } 30 \\
\mathrm{mg} \mathrm{2} \text { vezes ao dia }\end{array}$ \\
& & $5.700 \mathrm{UI} /$ dia \\
Nadroparina & $2.850 \mathrm{Ul} /$ dia & $5.700 \mathrm{UI} / \mathrm{dia}$ \\
Nadroparina & $2.850 \mathrm{Ul} / \mathrm{dia}$ & \\
\hline
\end{tabular}

${ }^{a}$ Todas s.c. 


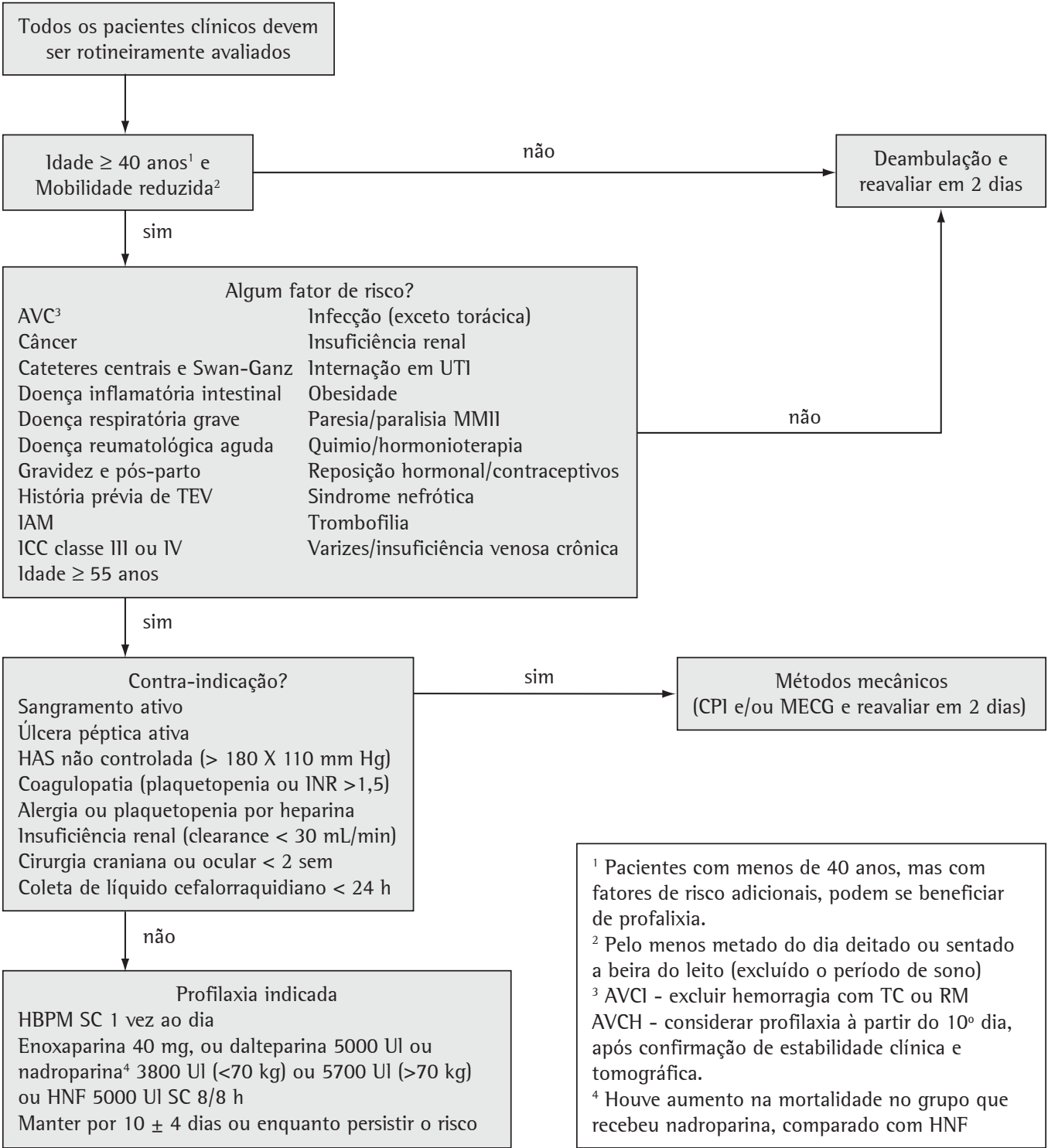

Figura 4 - Algoritmo para a avaliação do risco de TEV e profilaxia correspondente em pacientes clínicos. Retirado de estudos anteriores. ${ }^{(36-38)}$ ICC: insuficiência cardíaca congestiva; MMIl: membros inferiores; HAS: hipertensão arterial sistêmica; sem: semana; $\mathrm{AVCl}$ : AVC isquêmico; e AVCH: AVC hemorrágico.

laxia de TEV em pacientes submetidos à cirurgia oncológica abdominal ou pélvica, e mostrou resultados similares. $^{(23)}$ Um estudo complementar evidenciou que um regime de 4 semanas de profilaxia com enoxaparina, em comparação ao de 1 semana, reduz a ocorrência de TEV em cirurgias de neoplasia abdominal ou pélvica, sem alterar o sangramento. ${ }^{(24)}$ É interessante o uso combinado de métodos mecânicos de prevenção nesses pacientes. Em pacientes submetidos à cirurgia oncológica maior, sugerimos prolongar o tempo de profilaxia, que pode ser mantida inclusive após a alta hospitalar.(B)

A cirurgia por videolaparoscopia é um procedimento que tem sido cada vez mais empregado desde a década de 80 . As suas vantagens incluem menor trauma cirúrgico, menor permanência hospitalar e redução da dor e da imobilização pós-operatória. Tais achados podem sugerir enganosamente que o risco de TEV pósoperatória pode ser considerado mínimo em cirurgias laparoscópicas. Entretanto, alguns fatores próprios da videolaparoscopia tendem a 
aumentar o risco de trombose, como a pressão de insuflação peritoneal usada, que provoca venostase nos membros inferiores, e a hipercoagulabilidade induzida pelo pneumoperitônio. A posição de Trendelenburg invertida - posição supina em aclive - necessária para a exposição adequada do campo operatório, especialmente utilizada na colecistectomia videolaparoscópica, acentua a venostase e pode ser um fator que justifique a profilaxia diferenciada. ${ }^{(25)}$ Pacientes submetidos à videolaparoscopia sem fatores de risco adicionais para TEV necessitam apenas deambulação precoce. Aqueles com outros fatores de risco devem receber tromboprofilaxia (HNF, HBPM, MECG ou CPI).(B)

A TVP é uma causa importante de complicação da cirurgia para obesidade mórbida. Uma análise retrospectiva de prontuários de 5.554 pacientes submetidos à cirurgia bariátrica revelou 12 casos (0,21\%) de TEP fatal. ${ }^{(26)} \mathrm{Um}$ estudo prospectivo, no qual se avaliou 481 pacientes submetidos à cirurgia bariátrica que fizeram uso de MCEG e CPI e receberam enoxaparina (30 ou $40 \mathrm{mg}$ a cada $12 \mathrm{~h}$ ), demonstrou que o uso de $40 \mathrm{mg}$ de enoxaparina reduziu significativamente a incidência de TVP, sem diferenças de sangramento. ${ }^{(27)}$ As doses de heparina devem ser maiores que as usuais para pacientes não obesos (por ex., enoxaparina, $40 \mathrm{mg}$ duas vezes ao dia).(C)

Pacientes submetidos à cirurgia ortopédica maior, como artroplastia total de quadril ou de joelho, ou cirurgia de fratura de quadril, têm uma alta incidência de TEV, podendo atingir até $85 \%$ de TVP distal nas artroplastias de joelho na ausência de tromboprofilaxia. ${ }^{(5)}$ Nesses contextos, os pacientes devem receber HBPM (dose profilática alta) ou AVK (RN1 = 2-3). (A) A dabigatrana pode ser uma alternativa nessas circunstâncias.(B) 0 uso de HNF, MECG ou CPI isoladamente não é recomendado.(A) 0 regime de HBPM pode ser iniciado $12 \mathrm{~h}$ antes da cirurgia ou 12-24 $\mathrm{h}$ após, tendo como alternativa o início com a metade da dose profilática alta 4-6 $\mathrm{h}$ antes da cirurgia e atingindo a dose plena no dia seguinte.(A)

Um aspecto importante a ser considerado é a manutenção da profilaxia após a alta hospitalar. Do ponto de vista teórico, dever-se-ia permanecer utilizando as medidas preventivas enquanto persistissem os fatores de risco. Nos estudos clínicos, verificou-se que, para pacientes submetidos a cirurgias ortopédicas, deve-se manter a tromboprofilaxia até 35 dias após a alta.(A) ${ }^{(28)}$ Em pacientes que realizaram cirurgia oncológica, é sugerida utilização da profilaxia por pelo menos 28 dias após a alta hospitalar. $(A)^{(24)}$

Outros contextos cirúrgicos (cirurgia vascular, torácica, ginecológica e revascularização miocárdica) têm recomendações específicas, mas que são semelhantes às já mencionadas, variando com a especificidade de cada procedimento (risco de trombose e de sangramento). ${ }^{(5)}$

\section{Profilaxia de TEV em pacientes clínicos}

0 uso da tromboprofilaxia é bem estabelecido para a maioria dos pacientes cirúrgicos; porém, a situação para pacientes clínicos hospitalizados é menos conhecida, tendo em vista a grande heterogeneidade dessa população. ${ }^{(29)} \mathrm{A}$ profilaxia de TEV em pacientes clínicos é uma área na qual os possíveis benefícios para o paciente são grandes, já que TEP fatal é uma das mais comuns causas evitáveis de morte hospitalar, sendo resultado da subutilização de profilaxia nesses doentes. ${ }^{(30)}$

Pacientes agudamente doentes, hospitalizados e acamados, e que apresentem vários fatores de risco para TEV devem receber profilaxia.

Um estudo comparou o uso de HNF (5.000 UI a cada $8 \mathrm{~h}$ ) com o de enoxaparina (40 mg/dia) em pacientes clínicos internados com insuficiência cardíaca congestiva ou insuficiência respiratória grave. Os dois esquemas se mostraram efetivos e seguros. ${ }^{(31)}$ Outro estudo avaliou três regimes de profilaxia [placebo, enoxaparina (20 mg/dia) e enoxaparina ( $40 \mathrm{mg} / \mathrm{dia})] \mathrm{em}$ pacientes clínicos hospitalizados com pelo menos um fator de risco adicional para TEV (média, 2,1). 0 regime de enoxaparina de $40 \mathrm{mg} /$ dia significativamente reduziu o risco de TVP sem alterar a segurança. (32) A dose de enoxaparina de $20 \mathrm{mg} /$ dia não foi eficaz e não deve ser utilizada em pacientes clínicos. Outro estudo comparou o uso de dalteparina (5.000 UI/dia) ou placebo durante 2 semanas em 2.991 pacientes $>40$ anos de idade, com condição clínica aguda, hospitalização projetada para mais de 4 dias e pelo menos um fator adicional de risco. ${ }^{(33)} 0$ desfecho final mostrou que a prevenção com dalteparina reduziu significativamente a ocorrência de TEV sem aumentar o risco de sangramento maior. Uma meta-análise reunindo nove artigos 
comparando o uso de HNF e HBPM na tromboprofilaxia de pacientes clínicos $(n=4.669)$ demonstrou eficácia e segurança similares no uso dessas heparinas. ${ }^{(34)}$

Recomenda-se avaliar todos os casos de pacientes internados com doença clínica aguda, imobilização projetada para 3 ou mais dias e na presença de fatores de risco adicionais. $(A)^{(35)}$ Um grupo de trabalho da Associação Médica Brasileira, através de uma revisão sistemática da literatura, elaborou recomendações atuais de profilaxia para pacientes clínicos, que são apresentadas na Figura 4. ${ }^{(36,37)}$

A síndrome da classe econômica, inicialmente descrita em médicos que realizavam longas viagens aéreas e desenvolviam TEV, foi o primeiro indício que as viagens poderiam ser um fator de risco. Em um estudo avaliando um total de 135 milhões de passageiros, a incidência de TEP foi mais frequente em viagens maiores que $5.000 \mathrm{~km}-1,5$ casos por milhão de pessoas vs. 0,01 casos por milhão de pessoas em viagens mais curtas. ${ }^{(38)}$

Indivíduos em viagens aéreas $>8 \mathrm{~h}$ devem evitar roupas apertadas, manter hidratação adequada e fazer contrações frequentes das panturrilhas.(B) Pacientes que têm outro fator de risco adicional para TEV podem utilizar MECG (abaixo do joelho, com pressão de 15-30 mmHg no tornozelo) ou receber dose única de HBPM antes da partida do voo.(C)

Apesar da existência de evidências claras quanto aos benefícios da profilaxia de TEV em pacientes sob risco na redução de eventos tromboembólicos, o seu uso persiste negligenciado. Os estabelecimentos de saúde devem implementar estratégias para a disseminação do uso das medidas preventivas para TEV.(A) 0 uso de alertas eletrônicos para os médicos assistentes seria uma alternativa para aumentar o uso da profilaxia e, consequentemente, reduzir a taxa de TEV. Em um estudo randomizado e controlado, no qual foi avaliada a estratégia de alerta eletrônico para os médicos cujos pacientes hospitalizados não estivessem recebendo profilaxia para TEV, os resultados foram promissores. 0 alerta eletrônico foi eficaz em aumentar a prescrição da profilaxia de TEV no grupo intervenção e reduziu em 41\% o risco de TEV em 90 dias, em comparação com o grupo controle. ${ }^{(39,40)}$

\section{Referências}

1. Alikhan R, Peters F, Wilmott R, Cohen AT. Fatal pulmonary embolism in hospitalised patients: a necropsy review. J Clin Pathol. 2004;57(12):1254-7.

2. Yoo HH, Mendes FG, Alem CE, Fabro AT, Corrente JE, Queluz TT Clinicopathological findings in pulmonary thromboembolism: a 24-year autopsy study. J Bras Pneumol. 2004;30(5):426-32.

3. Goldhaber SZ, Dunn K, MacDougall RC. New onset of venous thromboembolism among hospitalized patients at Brigham and Women's Hospital is caused more often by prophylaxis failure than by withholding treatment. Chest. 2000;118(6):1680-4.

4. Schmidt B, Michler R, Klein M, Faulmann G, Weber C, Schellong S. Ultrasound screening for distal vein thrombosis is not beneficial after major orthopedic surgery. A randomized controlled trial. Thromb Haemost. 2003;90(5):949-54.

5. Geerts WH, Bergqvist D, Pineo GF, Heit JA, Samama CM, Lassen MR, et al. Prevention of venous thromboembolism: American College of Chest Physicians Evidence-Based Clinical Practice Guidelines (8th Edition). Chest. 2008;133(6 Suppl):381S-453S.

6. Geerts WH, Pineo GF, Heit JA, Bergqvist D, Lassen MR, Colwell CW, et al. Prevention of venous thromboembolism: the Seventh ACCP Conference on Antithrombotic and Thrombolytic Therapy. Chest. 2004;126(3 Suppl):338S-400S.

7. Amaragiri SV, Lees TA. Elastic compression stockings for prevention of deep vein thrombosis. Cochrane Database Syst Rev. 2000;(3):CD001484.

8. Urbankova J, Quiroz R, Kucher N, Goldhaber SZ. Intermittent pneumatic compression and deep vein thrombosis prevention. A meta-analysis in postoperative patients. Thromb Haemost. 2005;94(6):1181-5.

9. Charalambous C, Cleanthous S, Tryfonidis M, Goel A, Swindell R, Ellis D. Foot pump prophylaxis for deep venous thrombosis-rate of effective usage following knee and hip arthroplasty. Int Orthop. 2003;27(4):20810.

10. Kakkos SK, Caprini JA, Geroulakos G, Nicolaides AN, Stansby GP, Reddy DJ. Combined intermittent pneumatic leg compression and pharmacological prophylaxis for prevention of venous thromboembolism in high-risk patients. Cochrane Database Syst Rev. 2008;(4):CD005258

11. Obeid FN, Bowling WM, Fike JS, Durant JA. Efficacy of prophylactic inferior vena cava filter placement in bariatric surgery. Surg Obes Relat Dis. 2007;3(6):606-8; discussion 609-10.

12. Hovens MM, Snoep JD, Tamsma JT, Huisman MV. Aspirin in the prevention and treatment of venous thromboembolism. J Thromb Haemost. 2006;4(7):1470-5.

13. Prevention of venous thrombosis and pulmonary embolism. NIH Consensus Development. JAMA. 1986;256(6):744-9.

14. Clagett GP, Anderson FA Jr, Geerts W, Heit JA, Knudson M, Lieberman JR, et al. Prevention of venous thromboembolism. Chest. 1998;114(5 Suppl):531S560 .

15. King CS, Holley AB, Jackson JL, Shorr AF, Moores LK. Twice vs three times daily heparin dosing for thromboembolism prophylaxis in the general medical 
population: A metaanalysis. Chest. 2007;131(2):50716.

16. Kanaan AO, Silva MA, Donovan JL, Roy T, Al-Homsi AS. Meta-analysis of venous thromboembolism prophylaxis in medically 111 patients. Clin Ther. 2007;29(11):2395405.

17. Wein L, Wein S, Haas SJ, Shaw J, Krum H. Pharmacological venous thromboembolism prophylaxis in hospitalized medical patients: a meta-analysis of randomized controlled trials. Arch Intern Med. 2007;167(14):147686.

18. Mismetti P, Laporte S, Zufferey P, Epinat M, Decousus $\mathrm{H}$, Cucherat M. Prevention of venous thromboembolism in orthopedic surgery with vitamin $\mathrm{K}$ antagonists: a meta-analysis. J Thromb Haemost. 2004;2(7):1058-70.

19. Bergqvist D. Low-molecular-weight heparin for the prevention of postoperative venous thromboembolism after abdominal surgery: a review. Curr Opin Pulm Med. 2005;11(5):392-7.

20. Warkentin TE, Levine MN, Hirsh J, Horsewood P, Roberts RS, Gent M, et al. Heparin-induced thrombocytopenia in patients treated with low-molecular-weight heparin or unfractionated heparin. $\mathrm{N}$ Engl J Med. 1995;332(20):1330-5.

21. Agnelli G, Piovella F, Buoncristiani P, Severi P, Pini $\mathrm{M}$, D'Angelo A, et al. Enoxaparin plus compression stockings compared with compression stockings alone in the prevention of venous thromboembolism after elective neurosurgery. N Engl J Med. 1998;339(2):80-5.

22. O’Donnell M, Weitz Jl. Thromboprophylaxis in surgical patients. Can J Surg. 2003;46(2):129-35.

23. Efficacy and safety of enoxaparin versus unfractionated heparin for prevention of deep vein thrombosis in elective cancer surgery: a double-blind randomized multicentre trial with venographic assessment. ENOXACAN Study Group. Br J Surg. 1997;84(8):1099-103.

24. Bergqvist D, Agnelli G, Cohen AT, Eldor A, Nilsson PE, Le Moigne-Amrani A, et al. Duration of prophylaxis against venous thromboembolism with enoxaparin after surgery for cancer. N Engl J Med. 2002;346(13):97580.

25. Maciel R, Menna-Barreto SM. Thromboprofilaxis for videolaparoscopic cholecystectomy. J Bras Pneumol. 2004;30(5):480-4.

26. Sapala JA, Wood MH, Schuhknecht MP, Sapala MA. Fatal pulmonary embolism after bariatric operations for morbid obesity: a 24-year retrospective analysis. Obes Surg. 2003;13(6):819-25.

27. Scholten DJ, Hoedema RM, Scholten SE. A comparison of two different prophylactic dose regimens of low molecular weight heparin in bariatric surgery. Obes Surg. 2002;12(1):19-24.

28. Sapala JA, Wood MH, Schuhknecht MP, Sapala MA. Fatal pulmonary embolism after bariatric operations for morbid obesity: a 24-year retrospective analysis. Obes Surg. 2003;13(6):819-25.

29. Comissão de Circulação Pulmonar da Sociedade Brasileira de Pneumologia e Tisiologia. Recomendações para a prevenção do tromboembolismo venoso. J Pneumol. 2000;26(3):153-8.

30. Goldhaber SZ, Turpie AG. Prevention of venous thromboembolism among hospitalized medical patients. Circulation. 2005;111(1):e1-3.

31. Kleber FX, Witt C, Vogel G, Koppenhagen K, Schomaker $\mathrm{U}$, Flosbach CW, et al. Randomized comparison of enoxaparin with unfractionated heparin for the prevention of venous thromboembolism in medical patients with heart failure or severe respiratory disease. Am Heart J. 2003;145(4):614-21.

32. Samama MM, Cohen AT, Darmon JY, Desjardins L, Eldor A, Janbon C, et al. A comparison of enoxaparin with placebo for the prevention of venous thromboembolism in acutely ill medical patients. Prophylaxis in Medical Patients with Enoxaparin Study Group. N Engl J Med. 1999;341(11):793-800.

33. Leizorovicz A, Cohen AT, Turpie AG, Olsson CG, Vaitkus PT, Goldhaber SZ, et al. Randomized, placebocontrolled trial of dalteparin for the prevention of venous thromboembolism in acutely ill medical patients. Circulation. 2004;110(7):874-9.

34. Mismetti P, Laporte-Simitsidis S, Tardy B, Cucherat M, Buchmüller A, Juillard-Delsart D, et al. Prevention of venous thromboembolism in internal medicine with unfractionated or low-molecular-weight heparins: a meta-analysis of randomised clinical trials. Thromb Haemost. 2000;83(1):14-9.

35. Leizorovicz A, Mismetti P. Preventing venous thromboembolism in medical patients. Circulation. 2004;110(24 Suppl 1):IV13-9.

36. Academia Brasileira de Neurologia; Associação de Medicina Intensiva Brasileira; Federação Brasileira das Associações de Ginecologia e Obstetrícia; Sociedade Brasileira de Angiologia e de Cirurgia Vascular; Sociedade Brasileira de Cancerologia; Sociedade Brasileira de Cardiologia; et al. Tromboembolismo venoso: profilaxia em pacientes clínicos - parte 1. São Paulo: Associação Médica Brasileira, Conselho Federal de Medicina; 2005.

37. Academia Brasileira de Neurologia; Associação de Medicina Intensiva Brasileira; Federação Brasileira das Associações de Ginecologia e Obstetrícia; Sociedade Brasileira de Angiologia e de Cirurgia Vascular; Sociedade Brasileira de Cancerologia; Sociedade Brasileira de Cardiologia; et al. Tromboembolismo Venoso: Profilaxia em Pacientes Clínicos - Parte 11. São Paulo: Associação Médica Brasileira, Conselho Federal de Medicina; 2005.

38. Lapostolle F, Surget V, Borron SW, Desmaizières M, Sordelet D, Lapandry C, et al. Severe pulmonary embolism associated with air travel. $\mathrm{N}$ Engl J Med. 2001;345(11):779-83.

39. Kucher N, Koo S, Quiroz R, Cooper JM, Paterno MD, Soukonnikov B, et al. Electronic alerts to prevent venous thromboembolism among hospitalized patients. N Engl J Med. 2005;352(10):969-77.

40. Institute for Clinical Systems Improvement. Venous thromboembolism prophylaxis. Bloomington: Institute for Clinical System Improvement; 2009. 